\title{
Recent Advances in Wireless Localization Technologies
}

\author{
Shahrokh Valaee $\cdot$ Xinrong Li
}

Received: 23 October 2012/ Accepted: 25 October 2012/Published online: 6 November 2012

(C) Springer Science+Business Media New York 2012

Most wireless devices manufactured today are equipped with a plurality of sensors and communication capabilities. Handheld devices can nowadays connect through multiple communication networks, such as 3G/LTE, WiFi, Bluetooth, Zigbee, WiMax, UWB, etc. They are also capable of sensing the environment, taking still images or videos, finding their location, detecting hand movements via accelerometers, and reading RFID tags. These devices have advanced operating systems capable of sophisticated signal processing and wireless communications. Much research is needed to use the sensing and communication capabilities of the newest generation of wireless devices to build new applications such as real-time youtube or wikis that collect, process, and report live data to public for security, news, or entertainment purposes. Among the applications envisioned for the new technology are vehicular networks, assistive technologies, eHoming (smart home), and the entertainment industry.

The powerful capabilities of new smart phones and PDAs motivate new directions in academic research that seek to fully harvest the potential of advanced features for the creation of new network services. Most such emerging services will need the user location, which is not usually trivial to obtain. Interesting applications where location is an important factor for service enabling are assistive technologies, especially for navigation of visually impaired,

S. Valaee

Department of Electrical and Computer Engineering, University

of Toronto, Toronto, ON, Canada

e-mail:valaee@comm.utoronto.ca

X. Li $(\bowtie)$

Department of Electrical Engineering,

University of North Texas, Denton, TX, USA

e-mail: xinrong.li@unt.edu vehicular safety enhancement, event and situation-aware services, and so on. Despite much effort in recent years on location estimation both using active and passive methods, location estimation remains to be a challenging problem, especially in indoor environments where GPS signal is not available or the GPS location estimate is fairly inaccurate. This special issue tries to address some of the challenges in the location estimation and offer solutions where available.

The annual IEEE International Symposium on Personal, Indoor and Mobile Radio Communications (PIMRC) is one of the premier conferences in the wireless research arena and has a long history of bringing together academia, industry and regulatory bodies. Today, it has become one of the IEEE Communication Society's flagship conferences in telecommunications. The 22nd IEEE PIMRC was held successfully in Toronto, Canada, from September 11 to 14, 2011, where over 600 researchers met to share their latest research results in wireless technologies. In this special issue, we have compiled the extended versions of the best papers on localization from this conference as well as a few invited papers from well-known experts in this field. The invited authors have done an excellent job in substantially extending the conference version of the papers, which significantly improved the quality of the manuscripts. The objective of this special issue is to provide the readers a timely perspective on the exciting new developments in the field of wireless localization technologies.

The first paper, "A Comprehensive Tutorial on Localization: Algorithms and Performance Analysis Tools" by Davide Macagnano, Giuseppe Destino, and Giuseppe Abreu, was originally presented at PIMRC 2011 as a tutorial. The authors diligently converted the half-day tutorial presentation into a long tutorial article to provide a comprehensive view of technological solutions and theoretical fundamentals of localization and tracking (LT) systems for wireless 
networks. In this paper, localization and tracking systems are first classified into different categories and then differences among different types of systems are highlighted clearly. A wide variety of algorithms and techniques commonly used in localization and tracking systems are reviewed and the performance of these algorithms is compared through numerical simulations. Finally, the fundamental limits of localization technique are assessed through various performance lower bounds, including Cramer-Rao lower bound (CRLB), the Battacharyya Bound (BB), the Hammersley-ChapmannRobbins Bound (HCRB) and the Abel Hybrid Bound (AHB), in a source localization scenario.

The second paper is "Enhanced AoA Estimation and Localization through Transmitter Precoder Design" by Li Zhang, Yong Huat Chew, and Wai-Choong Wong". Angleof-arrival (AoA) estimation is a fundamental problem in many engineering applications, including wireless communications, radar, radio astronomy, sonar, navigation and tracking of objects. In this paper, the authors proposed a novel transmitter precoder-based algorithm to improve the accuracy in estimating AoA when the MUSIC (multiple signal classification) algorithm is used. An optimal precoder is first derived, which requires the channel side information at the transmitter and thus making it impractical for implementation. A more practical precoder design approach is then proposed, which utilizes the feedback CSIT estimated at the receiver. It is demonstrated through simulation results that the performance of the practical precoder matches the performance of the optimal precoder closely.

The third paper is "RF Localization for Wireless Video Capsule Endoscopy" by Kaveh Pahlavan, Guanqun Bao, Yunxing Ye, Umair Khan, Pranay Swar, Sergey Makarov, David Cave, Andrew Karellas, Prashant Krishnamurthy, and Kamran Sayrafian. The authors provided a comprehensive overview of an exciting new development in this field, that is, RF localization inside the human body. The first major application for such technology is the wireless video capsule endoscopy (VCE) that has been in the clinical arena for 12 years. RF localization-powered wireless VCE technology can help doctors to conveniently discover and locate medical problems inside the gastrointestinal (GI) tract without resorting to complex radiological or surgical operations. In this paper, the authors proposed a cyber-physical system (CPS) framework for experimental testing and visualization of interior of the human body that can be used for solving the RF localization problem for the endoscopy capsule.

The next paper is "High Resolution Cognitive RadioBased Concatenated Spectrum Time-of-Arrival Estimation" by Mohsen Pourkhaatoun and Seyed (Reza) Zekavat. The resolution of time-of-arrival (TOA) estimation is intrinsically determined by the signal bandwidth while the available spectrum is always limited in practice. In this paper, the authors proposed an interesting idea of virtually expanding signal bandwidth by concatenating non-contiguous and nonequal white spaces in the spectrum. White spaces in the spectrum may be identified and allocated by the capabilities of cognitive radios (CR). The authors designed various signal processing techniques to enable such spectrum concatenation for high-resolution TOA estimation. Performance of the proposed techniques is then studied through theoretical analysis of Cramer-Rao bounds and simulation results.

The last paper collected in this special issue is "Anonymous Indoor Navigation System on Handheld Mobile Devices for Visually Impaired" by Chen Feng, Shahrokh Valaee, Anthea Wain Sy Au, Sophia Reyes, Sameh Sorour, Samuel N. Markowitz, Deborah Gold, Keith Gordon, and Moshe Eizenman. In this paper, the authors proposed a received signal strength (RSS)-based indoor positioning and navigation system. The novelty of the proposed design is in the application of compressive sensing techniques in location estimation with a small number of noisy RSS measurements. A prototype indoor positioning and navigation system using the proposed algorithm is implemented on a PDA and experimental studies have been carried out in realistic application scenarios. The experimental results indicate that such a system can be readily deployed to assist visually impaired subjects to maneuver more conveniently in complex indoor environments.

At last, we would like to thank the authors of all special issue papers for their exceptional work in extending and improving their conference version papers to high-quality archival journal articles within a short period of time. We also thank the reviewers for their thoughtful, constructive comments, which have helped authors greatly to improve the quality of the papers. Last but not least, we would like to express our gratitude to the publishing editors and staff of Springer for their prompt assistance throughout the preparation of this special issue. We sincerely hope our readers enjoy reading the excellent papers presented in this special issue.

\section{Author Biographies}

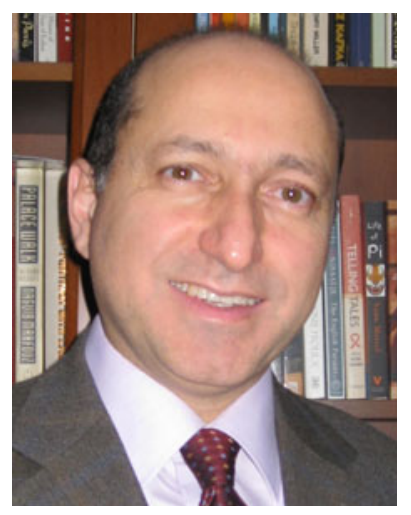

Shahrokh Valaee received the $\mathrm{PhD}$ degree in electrical engineering from McGill University in Canada. Currently he is a Professor and the Associate Chair for Undergraduate Studies and holds the Nortel Institute junior chair of Computer Networks in the Edward S. Rogers Sr. Department of Electrical and Computer Engineering at the University of Toronto. He is the founder and the Director of the Wireless and Internet Research Laboratory (WIRLab) at the University of Toronto. Prof. Valaee is an Editor of IEEE Transactions on Wireless Communications, and an Associate Editor of IEEE Signal Processing 
Letters. He was the Technical Program Co-Chair and the Local Organizing Chair of the IEEE PIMRC 2011, and the Co-Chair for Wireless Communications Symposium of IEEE GLOBECOM 2006. He has served as a guest editor for several journals including IEEE Wireless Communications Magazine, Wiley Journal on Wireless Communications and Mobile Computing, and EURASIP Journal on Advances in Signal Processing. His current research interests are in wireless vehicular and sensor networks, location estimation and cellular networks.

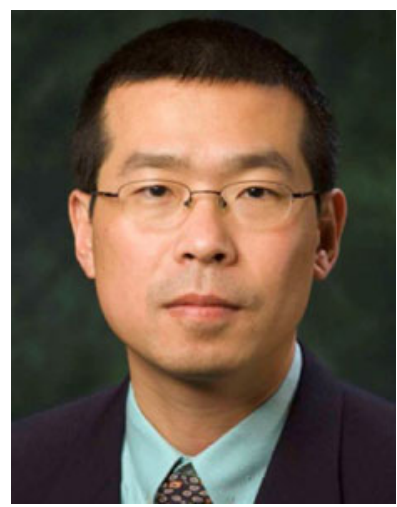

Dr. Xinrong Li received BE degree from the University of Science and Technology of China, Hefei, China, in 1995, ME degree from the National University of Singapore in 1999, and $\mathrm{PhD}$ degree from Worcester Polytechnic Institute (WPI), Worcester, MA, in 2003, all in Electrical Engineering. From 2003 to 2004, he was a Post-doc Research Fellow at the Center for Wireless Information Network Studies, WPI. He joined the Department of Electrical Engineering, University of North Texas, Denton, Texas, as an Assistant
Professor in 2004, and then he was tenured and promoted to Associate Professor in 2010. His recent research has been focused on statistical signal processing, geolocation, and wireless sensor network systems. 